\title{
On the Periodic Motions of Simple Hopping Robots
}

\author{
R.T. M'Closkey, J.W. Burdick, A.F. Vakakis
}

Mail Code 104-44, California Institute of Technology, Pasadena, CA, 91125

\section{Abstract}

This paper applies discrete dynamical systems theory to the analysis of simplified hopping robot models which are analogous to Marc Raibert's experimental machines. We first review a 1-dimensional vertical hopping model which captures both the vertical hopping dynamics and non-linear control algorithm. Second, we present a more complicated 2-dimensional model which includes both forward and vertical hopping dynamics and a foot placement algorithm. These systems are analyzed using a Poincaré return map, and hopping behavior is investigated by constructing the return map bifurcation diagrams with respect to system parameters. The bifurcation diagrams show a period doubling cascade leading to a regime of chaotic behavior. Using the vertical model results as a guide, we interpret the interesting dynamic behavior of the planar hopping system.

\section{Introduction}

Dynamically stable legged locomotion has attracted the interest of scientists and engineers since Muybridge and before. The work of Raibert and coworkers has certainly been among the most notable of the efforts to analyze and implement dynamically stable legged robot locomotion. Raibert [1] has developed a variety of theoretical and experimental models of of 1,2 , and 4 legged hopping machines which decompose locomotion into separate problems: the excitation of vertical motion, the maintenance of a desired forward running speed via a foot placement algorithm, and control of the body attitude.

In order to understand some of the interesting dynamical behavior, such as "limping gaits," seen in Raibert's experiments, Koditschek and Bühler [2] studied the dynamics of one-legged hopping from an analytical viewpoint. They developed a vertical hopping model which captures the essential non-linear features of Raibert's machines and uses a control algorithm analogous to Raibert's vertical excitation method. In addition to stable uniform vertical hopping motions, Koditschek and Bühler also demonstrated the existence of a stable period-2 hop which qualitatively resembled the experimental data of the limping gait.

Vakakis and Burdick [3] extended the Koditschek/Bühler model by relaxing the assumption used in [2] of an instantaneous thrust during the stance phase. In addition to corroborating the period-2 bifurcation seen in [2], the complete global bifurcation diagrams were constructed in [3] for cases of instantaneous and finite thrust duration. It was shown that a period-doubling cascades into chaos is possible, not just the period-2 hop predicted in [2]. It was also shown that the model in [2] was structurally unstable, meaning that global hopping behavior was substantially altered by a finite thrust duration. Thrust duration can be used to control the complexity of the chaotic attractor, and for sufficient large thrust duration, the attractor collapses to a globally stable uniform period-1 hopping motion.

The question remained as to whether the interesting behavio observed for vertical hopping would be preserved under lateral motion of the system. This paper extends Vakakis's model (Sec- tion 3) by including forward running dynamics and Raibert's foot placement algorithm. An analytical approximate Poincaré map is derived using the method of regular perturbation expansion (Section 4). An exact Poincaré map is also numerically computed (Section 5). The bifurcation diagrams of these maps (Section 6) show that the period doubling behavior observed in the 1-D model is preserved for non-zero forward velocities. In other words, Raibert's machines and leg placement algorithms are theoretically predicted to have period-2 and higher period stable hopping motions for some system parameter values, even for a foot placement algorithm which is intended to have only period-1 hopping motions. We suggest that the limping gait is likely due to the period doubling seen in our simplified models. These results demonstrate the importance of both the Poincaré map analysis and the predictive power provided by the simple vertical model. Because of length restrictions, interested readers should consult [5] for the more detailed derivations and results which are abbreviated in this paper.

\section{Review and Summary of Vertical Hopping Model and} Results

The salient features and behavior of the 1-D model in [3] are reviewed in this section, as this model provides insight into the more complex 2-D model considered in later sections. $[3,4]$ should be consulted for more in-depth analysis of the 1-D map. The simplified vertical hopping robot model consists of a point mass fixed to a massless leg and actuator combination (Figure 1). The actuator is a pneumatic cylinder with restoring force, $F_{s}$, proportional to:

$$
F, \sim \frac{1}{r}
$$

where $r$ is the length of the cylinder, or leg. The stiffness of the nonlinear spring may be adjusted by changing the supply pressure to the cylinder. This is equivalent to multiplying $F_{s}$ by a constant.

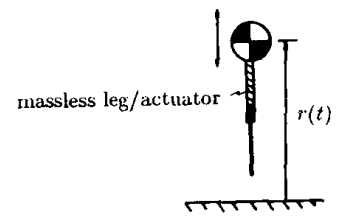

Figure 1: Vertical Hopping Robot Model

The vertical hopping cycle may be decomposed into four phases which are the 1-D analogy of Raibert's higher dimensional state machine models.

i. Thrust Phase. The $j^{\text {th }}$ hopping cycle begins at time $t_{j}$ when the leg reaches its maximum compression: $r_{j}=r\left(t_{j}\right)$, $\dot{r}\left(t_{j}\right)=0$. The control valves are opened and a constant supply pressure is applied to the leg cylinder for a fixed time, $\delta_{t}$. This results in a constant thrust force, $\tau$, which is the product of the supply pressure and the cross section of the pneumatic cylinder. The robot equation of motion during this phase is:

$$
\ddot{r}-\tau+g=0 \quad \text { for } t_{j} \leq t \leq t_{j}+\delta_{t}
$$

where $g$ is the gravitational constant. 
ii. Decompression Phase. At the end of the thrust phase, the valves are closed, defining an effective spring constant, $\eta_{2}=$ $\tau r_{e t}$, where $r_{e t}$ is the body position at the end of thrust phase. The equation of motion of the system during this phase is:

$$
\ddot{r}-\frac{\tau r_{e t}^{j}}{r}+g=0 \quad r_{e t} \leq r \leq r_{\max }
$$

where $r_{\max }$ is the uncompressed pneumatic cylinder reference length. The robot loses contact with the ground when it reaches the height $r_{\max }$

iii. Flight Phase. We assume that the lift-off and touchdown heights are identical to $r_{m a x}$, and that air drag during flight is negligible. The equation of motion in this phase is:

$$
\ddot{r}+g=0 \quad r \geq r_{\max }
$$

iv. Compression Phase. At touchdown, $\left(t_{t d}^{j}, r_{\max }\right)$, an initial pressure exists in the leg, fixing the spring constant during compression at $\eta$. 'The equation of motion during this phase

$$
\ddot{r}-\frac{\eta}{r}+g=0
$$

At the end of this phase, a new minimum height $r_{j+1}$ is reached at time $t_{j+1}$, and a new hopping cycle starts.

\subsection{Poincare Map for the 1 DOF Robot}

We are interested in analyzing the global nonlinear dynamic behavior of the 1 and 2-DOF hopping systems and the effect of system and control parameter variations on their behavior. Hopping is highly cyclic in nature, and a useful tool for analyzing non-linear systems with cyclic behavior is the Poincare return map [6]. This tool was used in [2] and [3] to analyze hopping behavior and will be used in this paper as well.

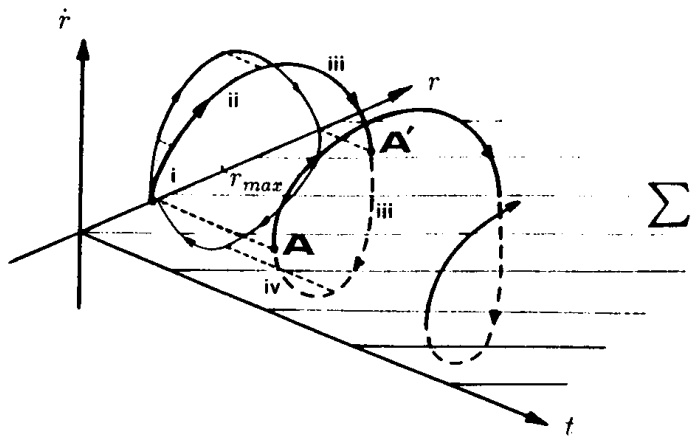

Figure 2: Phase space and Poincaré section

Given a set of initial conditions, equations (2.1)-(2.5) determine the motion of this dynamical system through four subsequent phases of a complete hopping cycle, and the phase space of the system is 3-dimensional: $(r, \dot{r}, t)$. The Poincaré Section, $\sum$, is a hyperplane in the extended phase space that intersects the trajectory of the system transversally (Figure 2). The Poincaré Return Map, which relates one point of intersection on $\sum$ to the next intersection point with the same orientation, has the form (for the 1-DOF system):

$$
r_{j+1}=f\left(r_{j}, t_{j}\right) ; \quad t_{j+1}=g\left(r_{j}, t_{j}\right) .
$$

In this case, let us locate $\Sigma$ at $\dot{r}=0$. The trajectory of the system will pierce $\Sigma$ transversally if $\ddot{r} \neq 0[3]$, which is satisfied whenever the robot is in motion. The tra jectory will pierce $\Sigma$ at two distinct points during each hopping cycle, denoted by $A$ and $A^{\prime}$ (Figure 2), which respectively correspond to the minimum and maximum hopping height. In [3], point $A$ was chosen as the location at which the dynamics were sampled, and it was shown that the vertical hopping return map reduced to a 1-dimensional map relating the minimum height of successive hops.

A detailed derivation of the 1-DOF model return map can be found in [3]. In deriving the Poincaré map, it was assumed that gravity forces during the stance phase are negligible compared to thrust and stiffness forces. This same assumption was made in [2]. The effects of including gravity during the stance phase is analyzed in [4], and will be briefly considered in Section 7. In brief, the return map is determined by integrating equations (2.1)-(2.5) over their respective intervals, using as initial conditions the final conditions of the previous phase. Introducing the non-dimensional leg length, $\omega_{j}$, and non-dimensional parameters $\lambda$ and $\beta$ :

$$
\omega=\frac{r_{j}}{r_{\max }} ; \quad \lambda=\frac{\tau r_{\max }}{\eta} ; \quad \beta=\frac{\tau \delta_{t}^{2}}{2 r_{\max }},
$$

the $1-\mathrm{D}$ return map has the non-dimensional form [3]:

$$
\omega_{j+1}=e^{\left(-\lambda \beta+\left(\lambda \beta+\lambda \omega_{j}\right) \ln \left(\beta+\omega_{j}\right)\right.} .
$$

The return map analysis has two important features: it reduces the dimension of the dynamics by one, and it converts the study of the continuous hopping robot dynamics to the analysis of a discrete nonlinear mapping (2.6). The fixed points, or period 1 orbits, of the return map correspond to motion which is uniform from bounce to bounce. Similarly, a period- $n$ orbit corresponds to a robot motion which repeats itself after $n$ hops. The stability of the periodic motions is determined by the stability of the return map periodic orbits, and an analysis of the 1-D map fixed point stability can be found in [3].

The main feature of this map is the period doubling bifurcations which occur as $\lambda$ is increased and $\beta$ is held constant. The bifurcation is termed co-dimension one because only one possible bifurcation variable is varied. Figure 3 shows the bifurcation diagram for $\beta=0$ (instantaneous thrust assumption). Increasing $\lambda$ can be physically accomplished by increasing the ratio of stance thrust force, $\tau$, to nonlinear spring constant, $\eta$. Physically, as $\lambda$ is increased beyond a certain critical value, the stable period one motion changes into a stable period two hop, which is interpreted as a repeating "two step". Further increases in $\lambda$ can lead to a period doubling cascade to chaos.

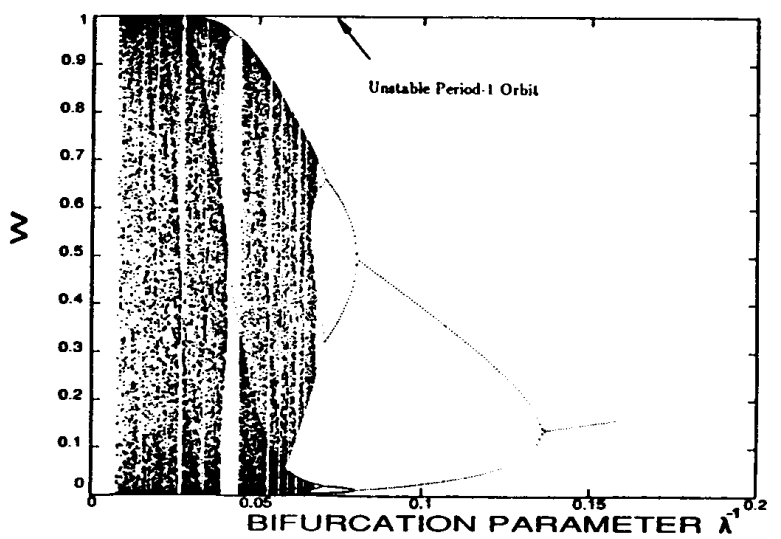

Figure 3: Global Bifurcation Diagram, case $\beta=0$

An interesting property of this map is the ability of nonzero $\beta$ (finite thrust time) to control the period doubling behavior [3]. Increasing $\beta$ decreases the complexity of the global dynamic be- 
havior, and for $\beta$ greater than a critical value, the period doubling behavior collapses to a globally stable period-1 hopping motion. A representative bifurcation diagram for $\beta=0.001$ is shown in Figure 4.

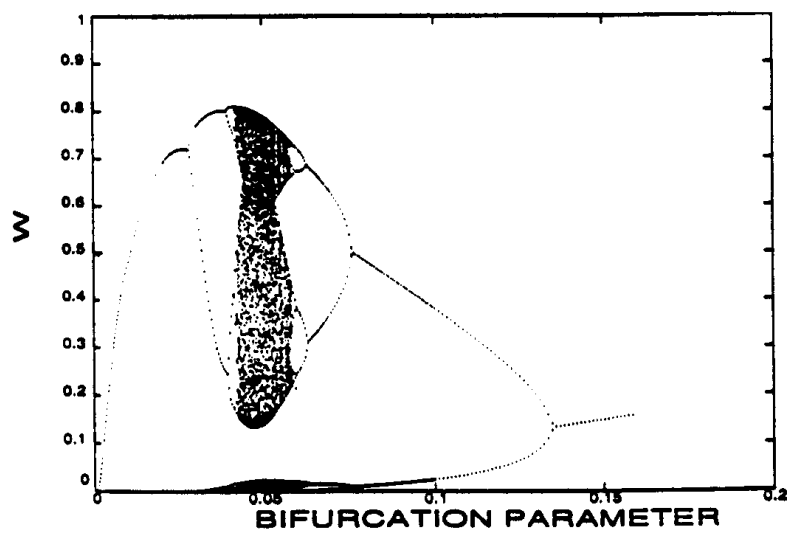

Figure 4: Global Bifurcation Diagram, case $\beta=0.001$

With the 1-D model as background, we proceed to introduce and analyze a more complete model which includes both vertical and horizontal hopping dynamics.

\section{The 2 DOF Model}

The total system mass is modeled as a point mass with no rotational inertia. The leg actuator and energy storage mechanis $m$ is an air cylinder with restoring force proportional to the inverse of the piston displacement, like the 1-DOF model. The leg and cylinder combination are assumed to be massless. Accordingly, only two independent coordinates are necessary to describe the configuration of the robot. It will be convenient to work with a polar coordinate system when the robot is in contact with the ground and a Cartesian system for ballistic motion. Let $r$ denote the leg length (analogous to the piston length in the 1-DOF model) and $\theta$ denote the "hip angle" during stance (Figure 5).

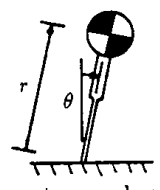

stance phase

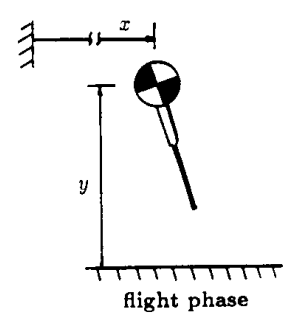

flight phase
Figure 5: Description of 2-DOF Hopping Model

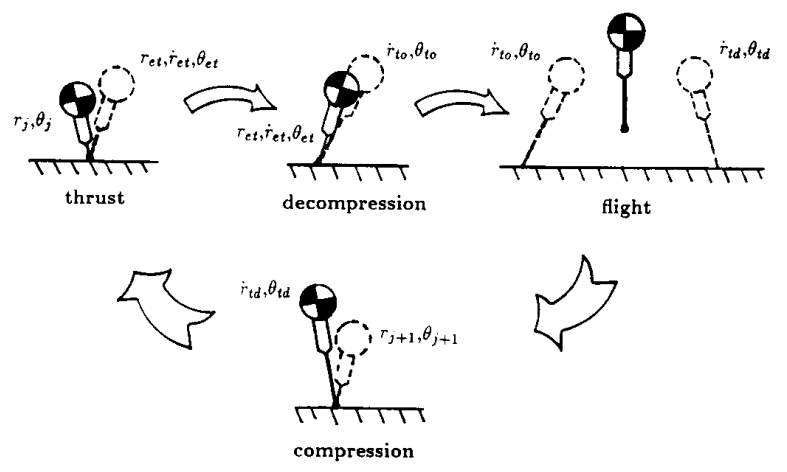

Figure 6: 2-DOF Hopping Robot Cycle
Like the 1-D model, the motion of the robot may be decomposed into four distinct phases : thrust, decompression, flight and compression (F'igure 6).

i. Thrust Phase. At time $t_{j}$ the leg is at its minimum radial length, $r_{j}=r\left(t_{j}\right)$ (i.e., $\dot{r}\left(t_{j}\right)=0$ ), the control valves are opened and a constant supply pressure is connected to the leg cylinder for a fixed time, $\delta_{t}$. This applies a constant radial force, $\tilde{\tau}$, to the mass. The equations of motion in this phase are:

$$
\begin{aligned}
& m\left(\ddot{r}-r \dot{\theta}^{2}\right)-\tilde{\tau}+m g \cos (\theta)=0 \quad \text { for } \quad t_{j} \leq t \leq t_{j}+\delta_{t} \\
& m(r \ddot{\theta}+2 \dot{r} \dot{\theta})-m g \sin (\theta)=0
\end{aligned}
$$

ii. Decompression Phase. At the end of the thrust phase the valves are closed and the cylinder decompresses. The effective spring constant is $\tilde{\tau}\left(r-r_{z e r o}\right)$, where $r_{z e r o}$ is the complementary piston length. The actual piston length is $r_{\max }-r_{\text {zero }}$. The piston length in the 1-DOF model does not matter because a "massless" extension may be added and the dimensions rescaled. The behavior of the 2-DOF model is affected because the change in $\theta$ is dictated by an angular momentum effect which becomes more pronounced as the mass approaches the foot pivot point. The spring force acts radially until the cylinder reaches length $r_{\max }$, which denotes the beginning of flight. The equations of motion are:

$$
\begin{aligned}
& m\left(\ddot{r}-r \dot{\theta}^{2}\right)-\frac{\tilde{\tau}\left(r_{e t}-r_{\text {zero }}\right)}{r-r_{z e r o}}+m g \cos (\theta)=0 \\
& m(r \ddot{\theta}+2 \dot{r} \dot{\theta})-m g \sin (\theta)=0 \quad r_{e t} \leq r \leq r_{\max }
\end{aligned}
$$

iii. Flight Phase. The final conditions of the decompression phase are converted from polar to Cartesian representation. Neglecting air drag during flight, the equations of motion are:

$$
\ddot{x}=0 ; \quad \ddot{y}+g=0 .
$$

The touchdown conditions at the end of flight are determined by the foot placement algorithm (FPA), which is discussed below.

iv. Compression Phase. At touchdown, the Cartesian variables are mapped to polar coordinates. The spring constant during this phase, $\tilde{\eta}$, is a parameter chosen by the designer. The end of the compression phase is signaled by the condition $\dot{r}=0$. The equations of motion are:

$$
\begin{aligned}
& m\left(\ddot{r}-r \dot{\theta}^{2}\right)-\frac{\tilde{\eta}}{r-r_{\text {zero }}}+m g \cos (\theta)=0 \\
& m(r \ddot{\theta}+2 \dot{r} \dot{\theta})-m g \sin (\theta)=0
\end{aligned}
$$

At the end of this phase a new minimum height is reached and the cycle repeats.

The impact of the leg with the ground causes no energy loss because in this model we assume the leg is massless. Furthermore no frictional losses occur during any phase of motion. The time during contact with the ground (thrust, decompression and compression phases) is collectively termed the stance phase.

In order to bring this model in line with the one DOF hopper, several additional assumptions are made:

i. Gravity is neglected in the radial equations of motion during stance. This implies that the radial spring and thrust forces dominate. This assumption is also made in the 1-DOF model, and $\S 7$ give numerical results for a model which includes gravity during stance.

ii. Gravity is also neglected in the angular equations of motion. The implications for the 2-DOF model are: if $\theta$ is "small" 
during stance (small forward velocity), the torque about the foot due to gravity has a small effect on the motion of the mass. Further, the robot motion is roughly symmetric about the vertical position, so if one is only interested in the net angle change during the stance phase, the gravity term approximately integrates to zero in this equation of motion.

The simplified equations may be normalized by dividing by the mass, $m$, and the maximum leg length, $r_{\text {max }}$. The nondimensional length is called $\xi$ (i.e. $\xi=\frac{r}{r_{\max }}$ and $\xi_{z e r o}=\frac{r_{\text {ziero }}}{r_{\max }}$ ).

Assumption (ii) results in a conservation of angular momentum expression: $\xi^{2} \dot{\theta}=\varepsilon$, where $\varepsilon$ represents the normalized angular momentum of the point mass about its foot position. Angular momentum arises due to our assumption that the robot pivots about the foot, which is assumed not to slip during the stance phase. The quantity $\varepsilon$ remains constant during each stance phase due to the omission of gravity from the angular equations of motion. The conservation equation may be substituted in the radial equations to yield a differential equation entirely in $\xi$. These simplified equations are listed below for each phase:

i. thrust phase:

$$
\ddot{\xi}-\frac{\varepsilon_{1}^{2}}{\xi^{3}}-\tau=0 ; \quad \xi^{2} \dot{\theta}=\varepsilon_{1} ; \quad \tau=\frac{\tilde{\tau}}{m r_{\max }}
$$

ii. decompression phase:

iii. flight:

$$
\ddot{\xi}-\frac{\varepsilon_{1}^{2}}{\xi^{3}}-\frac{\tau\left(\xi_{e t}-\xi_{z e r o}\right)}{\xi-\xi_{\text {zero }}}=0 ; \quad \xi^{2} \dot{\theta}=\varepsilon_{1} .
$$

$$
\bar{x}=0 ; \quad \ddot{y}+g=0 .
$$

iv. compression:

$$
\ddot{\xi}-\frac{\varepsilon_{2}^{2}}{\xi^{3}}-\frac{\eta}{\xi-\xi_{\text {zero }}}=0 ; \quad \xi^{2} \dot{\theta}=\varepsilon_{2} ; \quad \eta=\frac{\tilde{\eta}}{m r_{\max }}
$$

Since the constant stance angular momentum may vary from hop to hop, $\varepsilon_{1}$ and $\varepsilon_{2}$ respectively denote angular momentum during the thrust/decompression and next compression phases. Also note that the dynamical equations reduce to the 1-DOF model equations for purely vertical motion.

\subsection{The Foot Placement Algorithm (FPA)}

In contrast to the 1-DOF model, the 2-DOF model requires a foot placement algorithm to actively control and balance its forward motion. The FPA does not enter explicitly in the equations of motion because the leg is assumed massless, but it determines where the robot foot should be located at touchdown. This determines the initial conditions for the ensuing phases. A complete description of the FPA may be found in $[1,5]$. Given a desired forward velocity, $\dot{x}_{\text {desired }}$, the algorithm places the foot a distance

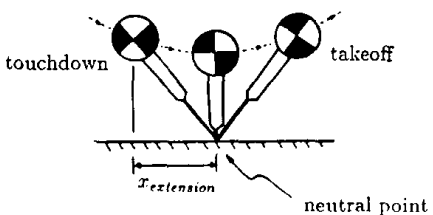

Figure 7: Description of Foot Placement Algorithm Variables

$x_{\text {extension }}$ in front of the body to regulate the actual forward velocity (Figure 7). $x_{\text {extension }}$ is computed as,

$$
x_{\text {extension }} \approx \frac{\dot{x} T_{\text {stance }}}{2}+\kappa_{\dot{x}}\left(\dot{x}-\dot{x}_{\text {desired }}\right)
$$

where $\dot{x}$ is the actual forward velocity, $\kappa_{\dot{x}}$ is a "gain" and $T_{\text {stance }}$ estimates the duration of the stance phase. This expression was developed for passive hopping without thrust and the factor of $\frac{1}{2}$ in the first term may be replaced by another gain, $\kappa_{\text {time }}$, to approximate for the effects of thrust. Note that the algorithm is largely kinematic in nature.

\section{The Poincaré Return Map for the 2 DOF Model}

During stance, the phase trajectory of the 2-DOF system is described by time, $t$, and the four states, $\xi, \dot{\xi}, \theta$ and $\dot{\theta}$. However, only $\xi, \dot{\xi}$ and $\theta$ are independent, as $\dot{\theta}$ is determined by conservation of angular momentum. Following [3], the dynamics of the system are sampled at the beginning of the thrust phase $(\dot{\xi}=0)$, and the return map will be independent of $\dot{\xi}$. It can also be shown that the system is autonomous, so that time can be ignored as well. The remaining two variables $\xi$ and $\theta$ do not fully describe the the dynamic behavior because the constant stance angular momentum may vary from hop to hop. Thus, the 2-DOF robot return map is three dimensional: $\xi, \theta$ and $\varepsilon$ are recorded at the start of each thrust phase. It should be noted that the three dimensional map reduces to the 1-D map for the case of purely vertical motion. Hence, the 1-D map is embedded in the three dimensional map.

The simplified equations ( 3.5 to 3.8 ) cannot be integrated to yield explicit functions of time. Consequently, a closed form Poincaré map is not obtainable, as for the 1-DOF model, and we must resort to other methods of constructing the return map. In this problem, direct numerical integration of the equations to derive the return map is not desirable for two reasons: (1) the nonlinear leg stiffness term is very sensitive to errors in $\xi$ and an adaptive time step, which is computationally burdensome, is required in order to maintain accuracy and stability of the algorithm; (2) valuable insight into the dynamics can be lost. For example, had the 1-D map of [3] been computed numerically, the dependence of the hopping dynamics on the physical parameters would not have been apparent.

Since an analytical return map is not feasible and direct numerical approaches have drawbacks, we seek an analytical approximation of the return map. A numerical method for finding the exact solution which does not rely upon brute force integration is discussed in $\S 5$. If we assume that $\varepsilon$ is "small," an approximate solution may be developed in terms of a regular perturbation series in $\varepsilon$. The perturbation solution must ultimately be verified with numerical solution of the equations, $\S 5$. The implication of a "small" $\varepsilon$ means that the robot moves forward with "small" velocity. No approximation is required for the flight phase equations.

\subsection{Perturbation Solution of the Equations}

The most elementary version of the perturbation method represents the solution of the simplified equations in the form of a power series in $\varepsilon$ :

$$
\begin{aligned}
& \xi\left(\varepsilon_{1}, t\right)=\xi^{(0)}(t)+\varepsilon_{1} \xi^{(1)}(t)+\varepsilon_{1}^{2} \xi^{(2)}(t)+\ldots \\
& \theta\left(\varepsilon_{1}, t\right)=\theta^{(0)}(t)+\varepsilon_{1} \theta^{(1)}(t)+\varepsilon_{1}^{2} \theta^{(2)}(t)+\ldots
\end{aligned}
$$

These expressions are substituted into the equations of motion and coefficients of powers of $\varepsilon_{1}$ are balanced. The final conditions of each phase will then have a power series representation which may be substituted into the initial conditions of the next phase. This process is carried out below in explicit detail in [5], and the results are summarized below. This perturbation expansion retains the first two non-trivial terms in each phase.

Thrust Phase Perturbation Solution. The radial thrust phase perturbation terms are (where the subscript et denotes "end of 


$$
\begin{aligned}
\text { thrust" }): & \\
\mathcal{O}(0): & \xi_{e t}^{(0)}=\frac{1}{2} \tau \delta_{t}^{2}+\xi_{0} ; \quad \dot{\xi}_{e t}^{(0)}=\tau \delta_{t} \\
\mathcal{O}(1): & \xi^{(1)}\left(\delta_{t}\right)=\xi_{0}^{(1)} ; \quad \dot{\xi}^{(1)}\left(\delta_{t}\right)=\dot{\xi}_{0}^{(1)} \\
\mathcal{O}(2): & \dot{\xi}_{e t}^{(2)}=\dot{\xi}_{0}^{(2)}+\frac{1}{\xi_{0}^{3}}\left[\frac{\delta_{t}}{4 \mu^{2}}+\frac{3 \delta_{t}}{8 \mu}+\frac{90}{\sqrt{\nu}} \tan ^{-1}\left(\sqrt{\nu} \delta_{t}\right)\right] \\
& \xi_{e t}^{(2)}=\xi_{0}^{(2)}+\frac{1}{\xi_{0}^{3} \frac{r}{2 \xi_{0}}}\left[\frac{\mu-1}{8 \mu}+\left(\frac{3}{16}-45\right) \ln \mu\right. \\
& \left.+90 \sqrt{\nu} \delta_{t} \tan ^{-1}\left(\sqrt{\nu} \delta_{t}\right)\right]
\end{aligned}
$$

where $\nu=\tau /\left(2 \xi_{0}\right)$ and $\mu=1+\mu \delta_{t}^{2}$; and the subscript 0 denotes the initial conditions of the hopping cycle. It can be shown that the first order initial conditions are actually zero. The angular perturbation coefficients are:

$$
\begin{aligned}
& \theta_{e t}^{(0)}=\theta_{0}^{(0)} \\
& \theta_{e t}^{(1)}=\theta_{0}^{(1)}+\frac{1}{\xi_{0}^{2}}\left[\frac{\delta_{t}}{\left(1+\frac{\tau}{2 \xi_{0}} \delta_{t}^{2}\right)}+\frac{1}{6} \delta_{t} \tan ^{-1}\left(\sqrt{\frac{\tau}{2 \xi_{0}}} \delta_{t}\right)\right]
\end{aligned}
$$

Decompression Phase Perturbation Solution. The end of decompression occurs when $\xi=1$. The two term perturbation expansion of $\dot{\xi}_{t o}$ is (where the subscript to denotes "take off"):

$$
\begin{aligned}
\dot{\xi}_{t o} & =\sqrt{\dot{\xi}_{e t}^{(0)^{2}}+2 \tau\left(\xi_{e t}^{(0)}-\xi_{z e r o}\right) \ln \left(\frac{1-\xi_{z e r o}}{\xi_{e t}^{(0)}-\xi_{z e r o}}\right)} \\
& +\varepsilon_{1}^{2} \frac{1}{2} \frac{\left[2 \dot{\xi}_{e t}^{(0)} \dot{\xi}_{e t}^{(2)}+2 \tau \xi_{e t}^{(2)} \ln \left(\frac{1-\xi_{z e r o}}{\xi_{e t}^{(0)}-\xi_{z e r o}}\right)-2 \tau \xi_{e t}^{(2)}-1+\frac{1}{\xi_{e t}^{(0)^{2}}}\right]}{\sqrt{\dot{\xi}_{e t}^{(0)^{2}}+2 \tau\left(\xi_{e t}^{(0)}-\xi_{z e r o}\right) \ln \left(\frac{1-\xi_{z e r o}}{\xi_{e t}^{(0)}-\xi_{z e r o}}\right)}}
\end{aligned}
$$

The angular terms are:

$$
\begin{aligned}
& \theta_{t o}^{(0)}=\theta_{e t}^{(0)} \\
& \theta_{t o}^{(1)}=\theta_{e t}^{(1)}+\frac{1}{\tau} \int_{\left|\dot{\xi}_{e t}^{(0)}\right|}^{\left|\xi_{t o}^{(0)}\right|} \frac{\exp \left(\frac{x^{2}-\dot{\xi}_{e t}^{(0)^{2}}}{2 \tau\left(\xi_{e t}^{(0)}-\xi_{z e r o}\right)}\right) d x}{\left(\xi_{\text {zero }}+\left(\xi_{e t}^{(0)}-\xi_{z e r o}\right) \exp \left(\frac{\left.x^{2}-\dot{\xi}_{e t}^{(0)}\right)^{2}}{2 \tau\left(\xi_{e t}^{(0)}-\xi_{z e r o}\right)}\right)\right)^{2}}
\end{aligned}
$$

where (4.7) may be evaluated by suitable numerical routines.

Flight Phase Solution. The radial variables must be "unnormalized" to calculate the true velocity at take-off. The velocity of the point mass in the radial direction is $\dot{r}=\xi r_{m a x}$ and the velocity tangent to the angular motion is $r_{\max } \dot{\theta}=r_{\max } \varepsilon$ (since $\xi=1$ at take-off). The takeoff variables in Cartesian coordinates are:

$$
\begin{aligned}
& \dot{x}_{t o}=\left(-\varepsilon_{1} \cos \left(\theta_{t o}\right)-\dot{\xi}_{t o} \sin \left(\theta_{t o}\right)\right) r_{\max } \\
& \dot{y}_{t o}=\left(-\varepsilon_{1} \sin \left(\theta_{t o}\right)+\dot{\xi}_{t o} \cos \left(\theta_{t o}\right)\right) r_{\max }
\end{aligned}
$$

where $\dot{\xi}_{t o}=\dot{\xi}_{t o}^{(0)}+\varepsilon_{1}^{2} \dot{\xi}_{t o}^{(2)}+\cdots$ and $\theta_{t o}=\theta_{t o}^{(0)}+\varepsilon_{1} \theta_{t o}^{(1)}+\ldots$ Note that $\dot{x}_{t o}$ and $\dot{y}_{t o}$ cannot be computed exactly since only a finite number of terms in the perturbation series of $\dot{\xi}_{t o}$ and $\theta_{t o}$ are evaluated and substituted into these equations. The only force acting on the mass is the gravitational force, hence the motion of the mass is ballistic. The details of the flight phase calculations are not presented but may be found in [5].

The FPA determines $x_{\text {extension }}(3.9)$. This fixes $\theta_{t d}$, and the velocities, $\dot{x}_{t d}$ and $\dot{y}_{t d}$ (where the subscript $t d$ denotes "touch $d$ own"). A reverse transformation changes the Cartesian variables back into a polar representation:

$$
\begin{aligned}
\dot{\xi}_{t d} & =\frac{\sqrt{\dot{x}_{t d}^{2}+\dot{y}_{t d}^{2}}}{r_{\text {max }}} \cos \left(\frac{\pi}{2}-\tan ^{-1}\left(\frac{\dot{y}_{t d}}{\dot{x}_{t d}}\right)+\theta_{t d}\right) \\
\varepsilon_{2} & =-\frac{\sqrt{\dot{x}_{t d}^{2}+\dot{y}_{t d}^{2}}}{r_{\text {max }}} \sin \left(\frac{\pi}{2}-\tan ^{-1}\left(\frac{\dot{y}_{t d}}{\dot{x}_{t d}}\right)+\theta_{t d}\right)
\end{aligned}
$$

where $\varepsilon_{2}$ is the new angular momentum that remains fixed during the new stance phase.

Compression Phase Perturbation Solution. The two term perturbation expansion of $\xi_{e c}$ is:

$$
\begin{aligned}
\xi_{e c}= & \xi_{\text {zero }}+\left(1-\xi_{\text {zero }}\right) e^{\frac{-\xi_{0}^{2}}{2 \eta}} \\
& +\varepsilon_{2}^{2} \frac{1}{2 \eta} e^{-\frac{\dot{\xi}_{0}^{2}}{2 \eta}}\left(1-\xi_{\text {zero }}\right)\left(\frac{1}{\left(\xi_{\text {zero }}+\left(1-\xi_{\text {zero }}\right) e^{\left.-\frac{\dot{\xi}_{0}^{2}}{2 \eta}\right)^{2}}-1\right)}\right)
\end{aligned}
$$

where $e c$ denotes "end of compression." The first two nonzero terms in the hip angle perturbation series are:

$$
\begin{aligned}
& \theta_{e c}^{(0)}=\theta_{t d} \\
& \theta_{e c}^{(1)}=\frac{\left(1-\xi_{z e r o}\right)}{\eta} \int_{0}^{\left|\dot{\xi}_{t d}\right|} \frac{\exp \left(\frac{x^{2}-\dot{\xi}_{t d}}{2 \eta}\right)}{\left(\xi_{\text {zero }}+\left(1-\xi_{\text {zero }}\right) \exp \left(\frac{x^{2}-\dot{\xi}_{t d}}{2 \eta}\right)\right)^{2}} d x
\end{aligned}
$$

Computation of $T_{\text {stance }}$. The computation of $x_{\text {extension }}$ via the FPA requires the calculation of the stance time, $T_{\text {stance. }}$. The stance time for the current stance phase is not known so Raibert approximates $T_{\text {stance }}$ from the previous stance phase. An analytical first order approximation may obtained by using the first integrals of motion of the unperturbed equations. Details of this derivation are in [5].

$$
\begin{aligned}
T_{\text {stance }}= & \delta_{t}+\frac{\left(1-\xi_{\text {zero }}\right)}{2 \eta} \int_{0}^{\left|\dot{\xi}_{t d}^{(0)}\right|} \exp \left(\frac{x^{2}-\dot{\xi}_{t d}^{2}}{2 \eta}\right) d x \\
& +\frac{1}{\tau} \int_{\left|\dot{\xi}_{e t}^{(0)}\right|}^{\left|\dot{\xi}_{t o}^{(0)}\right|} \exp \left(\frac{y^{2}-\dot{\xi}_{e t}^{(0)^{2}}}{2 \tau\left(\xi_{\text {et }}^{(0)}-\xi_{\text {zero }}\right)}\right) d y
\end{aligned}
$$

where $x^{2}=\dot{\xi}_{t d}^{(0)^{2}}+2 \eta \ln \left(\frac{\xi-\xi_{\text {erro }}}{1-\xi_{\text {zero }}}\right)$ and $y^{2}=\dot{\xi}_{e t}^{(0)^{2}}+2 \tau\left(\xi_{e t}-\right.$ $\left.\xi_{\text {zero }}\right) \ln \left(\frac{\xi-\xi_{\text {zero }}}{\xi_{\text {et }}-\xi_{\text {zero }}}\right)$.

The Poincaré return map is obtained by successively substituting the individual solutions of each phase into the following phase. However, the resulting size of the expressions makes this inconvenient, and we resort to a numerical analysis of this map, which is guided by results of the 1-D model. Analysis of the map properties, like that performed in [3] is possible, but our immediate goal is to determine whether the bifurcations seen in the 1-D map are also a feature of the forward running system. It is also easily seen how the 3-D map reduces to the 1-D map as $\varepsilon \rightarrow 0$.

\section{Exact Solution of the Equations}

We briefly outline an exact numerical scheme for determining the Poincaré map which combines numerical integration, quadrature and solution of transcendental equations. The advantage of this method over brute force numerical integration of the equations is the fact that the singularity problems which occur in numerically 
integrated solution do not arise here. The compression, thrust and decompression phases posses useful first integrals of motion for the radial variable. The thrust phase equations are integrated numerically with any suitable algorithm.

The velocity at take-off may be computed directly from the decompression first integral:

$$
\dot{\xi}_{t o}=\sqrt{\dot{\xi}_{e t}^{2}+2 \tau\left(\xi_{e t}-\xi_{z e r o}\right) \ln \left(\frac{1-\xi_{\text {zero }}}{\xi_{e t}-\xi_{\text {zero }}}\right)-\varepsilon_{1}^{2}\left(1-\frac{1}{\xi_{e t}^{2}}\right)} .
$$

Note that $\varepsilon$ is still the normalized angular momentum, but no assumption is made on its magnitude. The change in stannce angle may be computed from the following integral:

$\theta_{t o}=\theta_{e t}+\int_{\xi_{e t}}^{1} \frac{\varepsilon_{1} d \xi}{\xi^{2} \sqrt{\dot{\xi}_{e t}^{2}+2 \tau\left(\xi_{e t}-\xi_{z e r o}\right) \ln \left(\frac{\xi-\xi_{z e r o}}{\xi_{e t}-\xi_{\text {zero }}}\right)-\varepsilon_{1}^{2}\left(\frac{1}{\xi^{2}}-\frac{1}{\xi_{e t}}\right)}}$

The flight phase computations remain the same and $\xi_{e c}$ at the end of the compression phase is the solution of the following transcedental equation:

$$
-\frac{1}{2} \dot{\xi}_{t d}^{2}-\eta \ln \left(\frac{\xi_{e c}-\xi_{\text {zero }}}{1-\xi_{z e r o}}\right)+\frac{1}{2} \varepsilon_{2}^{2}\left(\frac{1}{\xi_{e c}^{2}}-1\right)=0
$$

$\theta_{e c}$ is computed using:

$$
\theta_{e c}=\theta_{t d}+\int_{\xi_{e c}}^{1} \frac{\varepsilon_{2}}{\xi^{2} \sqrt{\dot{\xi}_{t d}^{2}+2 \eta \ln \left(\frac{\xi-\xi_{z e r o}}{1-\xi_{\text {zero }}}\right)-\varepsilon_{2}^{2}\left(\frac{1}{\xi^{2}}-1\right)}} d \xi
$$

Expressions (5.2) to (5.4) may be evaluated to any desired accuracy using suitable numerical techniques.

\section{Results and Numerical Analysis of the 3-D Map}

While the exact solution in the previous section is useful for constructing accurate bifurcation diagrams, the perturbation solution demonstrates quantitatively that:

- The radial zeroth order terms of each phase may be combined to yield the 1-D return map of [3] for the case $r_{z e r o}=0$. Thus, the 1-DOF model should be a good qualitative predictor of the dynamic behavior of the 2-DOF model radial component.

- the FPA has a only second order effect on the radial portion of the map dynamics.

- the angle of the robot is determined by the FPA to zeroth order since $\theta_{t d}$ is directly determined by the FPA through the calculation of $x_{\text {extension. }}$. The change in angle during the stance phase derives from a first order effect.

The 1-D map is a function of two non-dimensional variables, $\lambda$ and $\beta$, and [3] studied co-dimension 1 bifurcations in which $\lambda$ is varied while $\beta$ is constant. The 2-DOF model has more parameters, $k_{\text {time }}$ and $k_{\dot{x}}$, which are candidate variables for bifurcation diagrams. The authors felt that the FPA gains should be chosen to yield stable behavior over the largest possible range of $\tau, \delta_{t}$, and $\eta$. Hence, only $\lambda$, was varied in our numerical investigation, as these results may be meaningfully compared to the co-dimension 1 bifurcations of the 1-D map. Note that variations in $\lambda$ are effected by changing the ratio $\tau$ to $\eta$. However, while increasing $\tau$ with fixed $\eta$ has the same qualitative effect as decreasing $\eta$ with fixed $\tau$, the robot will hop higher in the first case.

The radial motion is expected to exhibit period doubling behavior because the zeroth order terms in the approximate Poincaré map are exactly the 1-D map. There are no analogous 1-D map results for $\theta$ and $\varepsilon$ so their behavior must be determined by iteration of the map. Numerical generation of the the bifurcation diagrams follows [3]. Since the bifurcation diagram with respect to $\lambda$ is 3-dimensional, in Figures $8(\mathrm{a}, \mathrm{b}, \mathrm{c})$ we present the projections of the 3-D bifurcation diagram onto the $\xi, \theta$, and $\varepsilon$ axes. The exact and perturbation solutions are superimposed in each diagram. For this example, $\delta_{t}=0.01$ seconds, $\tau=15$, and $\eta$ varies from 1.5 to 0.2 . $\dot{x}_{\text {desired }}=0.1 \mathrm{~m} / \mathrm{sec}, k_{\text {time }}=0.4$, and $k_{\dot{x}}=0.1$. Figure 8 clearly illustrates: (1) how close the perturbation solution is to the exact solution; (2) that period doubling and reverse period doubling phenomena exist in this model as well. The bifurcation diagram is not complete because at high values of $\lambda$ the robot does not achieve enough height during the flight phase to place the foot where the FPA dictates. In other words, the robot "stubs its toe."

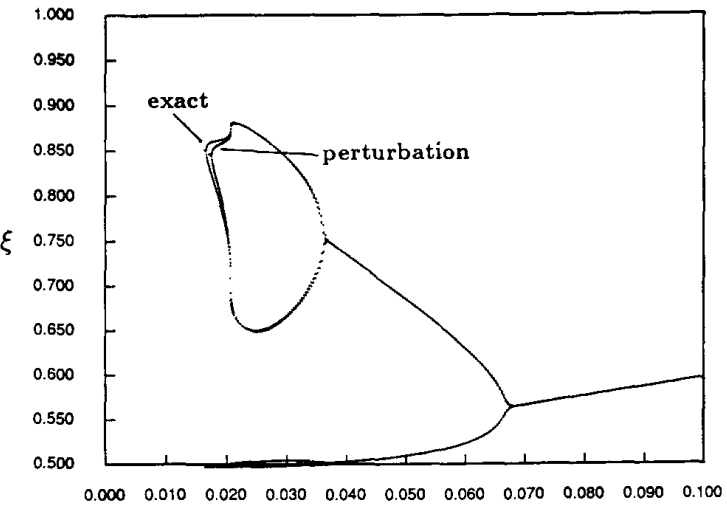
$\lambda^{-1}$
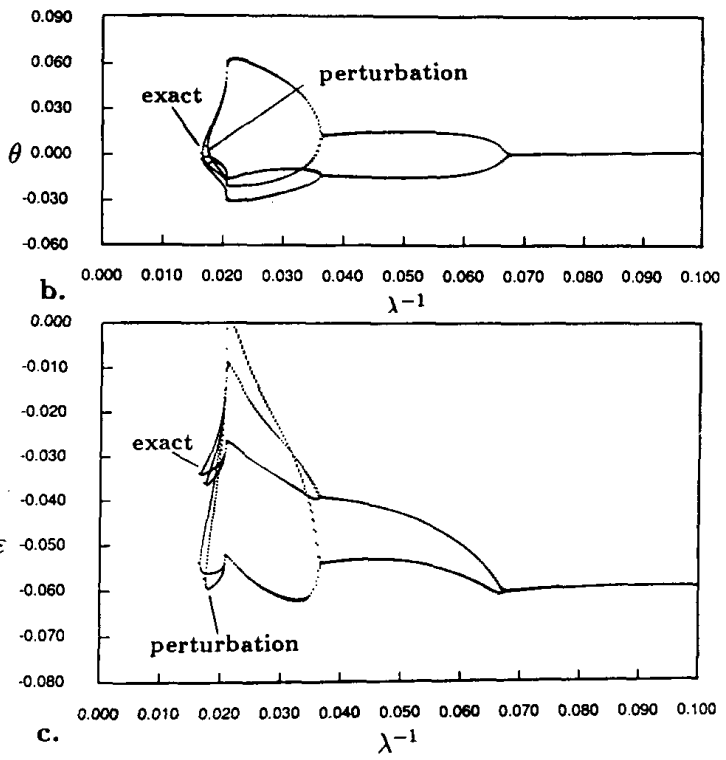

Figure 8: Bifurcation Diagram Components

To illustrate the effect of variations in $\beta$ on bifurcation behavior, the bifurcation diagrams in Figures $9(a, b, c)$ have the same parameters as Figure 8, except $\delta_{t}=0.005$. Figure 9 clearly shows the period doubling route to chaos first seen in the 1-DOF model. The perturbation and exact solutions are almost indistinguishable. Experience has shown that the perturbation and exact solutions exhibit the same qualitative behavior for $|\varepsilon|<0.2$

Figure 10 shows the strange attractor for the same parameters in Figure 9 with the specific value $\eta=0.375$. In other words, Figure 

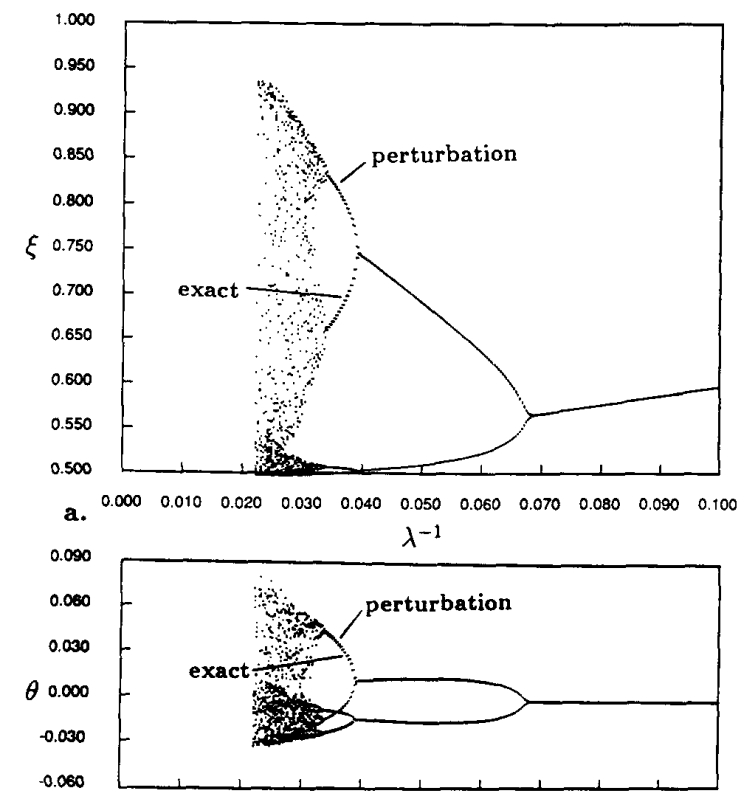

b. $\begin{array}{lllllllllll}0.000 & 0.010 & 0.020 & 0.030 & 0.040 & 0.050 & 0.060 & 0.070 & 0.090 & 0.090 & 0.100\end{array}$ b. $\lambda^{-1}$

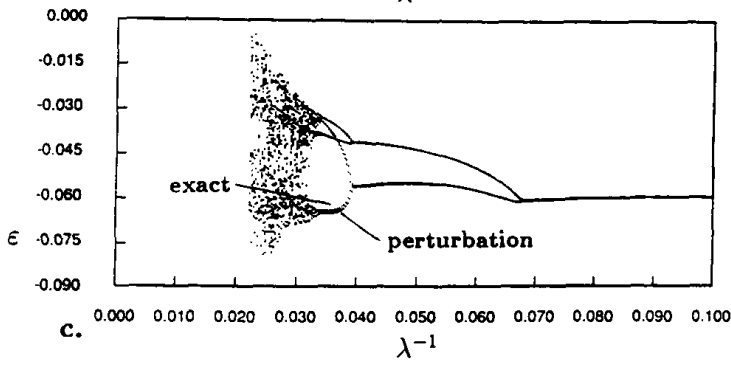

Figure 9: Bifurcation Diagram Components

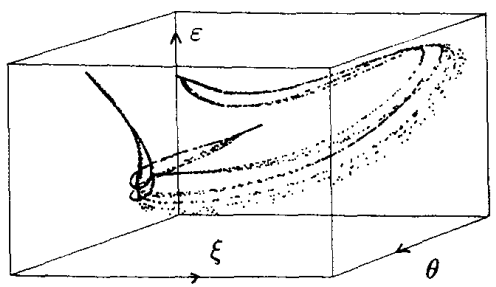

Figure 10: Strange Attractor in Hopping Dynamics

10 shows the attracting set of the hopping dynamics for a regime of chaotic behavior. [5] should be consulted for further simulations and bifurcation diagrams which qualitatively illustrate the effect of variations in forward running speed on dynamic behavior. Interestingly, the 3-D map can bifurcate to chaos for the same set of parameters which result in period-4 hopping in the 1-DOF model - the forward motion has an observable perturbative effect on the vertical hopping dynamics.

\section{Simulations with Gravity Included}

Does the period doubling observed above persist when gravity terms are included during the stance phase? A model with gravity implemented in all phases of motion is obviously a "better" model of the real system. The effect of gravity on the 1-DOF model is discussed in [4]. For the 2-DOF model, this question can not be currently answered with confidence. Figure 11 shows a plot of robot height vs. time for a simulation of a 2-DOF model including gravity. This simulation clearly illustrates that period-2 behavior is possible. The period doubling parameter values for this simulation were predicted directly from the 1-D map with gravity, much the same as the 1-DOF gravityless model closely predicts the period doubling bifurcation of the 2-DOF gravityless model.

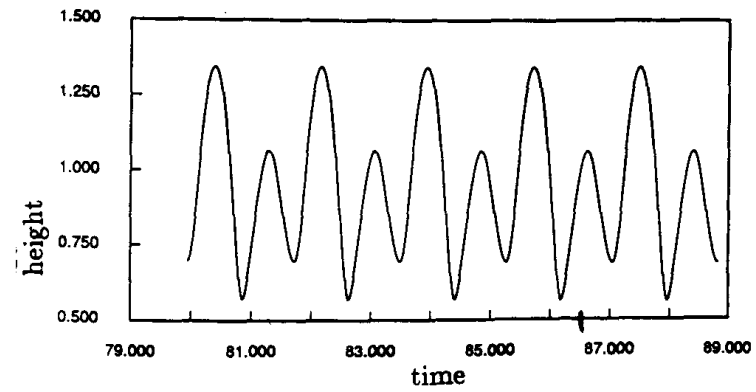

Figure 11: Robot Height During Period-2 Hop for 2-DOF 8. Conclusion

Model with Gravity

This paper developed a 2-DOF simplified model of Raibert's hopping robots which is an extension of previously developed vertical models [3]. In both the 1-DOF and 2-DOF models, the global dynamic behavior and its variation with respect to system and control parameter changes are analyzed via Poincaré return maps. Both an analytical approximate and an exact numerical return map were developed. The approximate solution, shown to be accurate for small forward velocity, quantitatively and qualitatively describes the coupling between the lateral and vertical dynamics and the effect of the FPA. Since the 1-D map developed in [3] is embedded in the 2-DOF model return map, we predicted that period doubling behavior should occur, as in the 1-DOF model. The bifurcation diagrams indeed verify that period doubling and chaotic behavior also occurs in the 2-DOF model. Further, we presented simulations which include gravity during stance phase which also exhibit period doubling behavior.

This analysis shows that exotic behavior can exist for such simple models, and is a further demonstration that the Poincare map is a useful tool for analyzing dynamically stable locomotion. This analysis suggests that Raibert's foot placement algorithms nominally lead to stable period-1 hopping motion, but can exhibit higher period stable hopping for some system parameter values. We propose that the perturbation model developed in this paper will be useful in future research on new foot placement algorithms.

\section{References}

[1] M. H. Raibert, Legged Robots that Balance, The MIT Press, Cambridge, Mass., 1986.

[2] D. Koditschek and M. Bühler, "Analysis of a Simplified Hopping Robot," Report no. 8804, Dept. of Electrical Engineering, Yale University, 1988.

[3] A.F. Vakakis, J.W. Burdick, "Chaotic Motions of a Simplified Hopping Robot," Proceedings of the IEEE International Conference on Robotics and Automation, Cincinnati, Ohio, May 14-18, 1990, pp. 1464-1469.

[4] A.F. Vakakis, R.T. M'Closkey, J.W. Burdick, "Study of a One-Dimensional Map Describing the Vertical Dynamics of a Hopping Robot, Robotics and Mechanical Systems Report no. RMS-90-01, School of Engineering and Applied Science, California Institute of Technology, in preparation.

[5] R.T. M'Closkey, J.W. Burdick, "The Periodic Motions of a Hopping Robot with Vertical and Forward Motion," Robotics and Mechanical Systems Report no. RMS-90-05, School of Engineering and Applied Science, California Institute of Technology, September, 1990.

[6] J. Guckenheimer and P. Holmes, Nonlinear Oscillations, Dynamical Systems and Bifurcations of Vector Fields, Springer Verlag, New York, 1983. 